\title{
Phase Transitions in Liquid Crystal Doped with Magnetic Particles of Different Shapes
}

\author{
P. Kopčanský • N. Tomašovičová · M. Koneracká • \\ M. Timko • V. Závišová • A. Džarová · J. Jadzyn • \\ E. Beaugnon - X. Chaud
}

Received: 1 April 2010 / Accepted: 28 June 2010 / Published online: 13 July 2010

(C) The Author(s) 2010. This article is published with open access at Springerlink.com

\begin{abstract}
In this study, observations of structural transitions in ferronematics based on the thermotropic nematic 4-trans-4'-n-hexyl-cyclohexyl-isothiocyanato-benzene (6CHBT) are described. Droplets of the nematic phase in the isotropic phase were observed in solutions of nematogenic 6CHBT dissolved in phenyl isocyanate and 6 CHBT dissolved in phenyl isocyanate and doped with magnetic particles of different shapes (nanorods and chain-like particles). Magneto-dielectric measurements of structural transitions in these new systems enable to estimate of the type of anchoring of the nematic molecules on the magnetic particles surface.
\end{abstract}

Keywords Ferronematics $\cdot$ Liquid crystal $\cdot$ Magnetic particles $\cdot$ Phase transition

\section{Introduction}

Liquid crystals possess the anisotropy of the physical properties that allow changes of the orientation of the liquid crystal optical axis (named director $\boldsymbol{n}$ ) under the influence of external electric or magnetic fields. In restricted geometry this effect is called the Fréedericksz transition. The classical Fréedericksz transition has been well studied experimentally and theoretically [1]. However, due to the small anisotropy of the

P. Kopčanský · N. Tomašovičová $(\bowtie) \cdot$ M. Koneracká · M. Timko · V. Závišová · A. Džarová Institute of Experimental Physics, Slovak Academy of Sciences, Watsonova 47, 04353 Košice, Slovakia e-mail:nhudak@saske.sk

\section{J. Jadzyn}

Institute of Molecular Physics, Polish Academy of Sciences, Smoluchowskiego 17, 60179 Poznan, Poland

E. Beaugnon $\cdot$ X. Chaud

Grenoble High Magnetic Field Laboratory, Centre National de la Recherche Scientifique,

25 Avenue des Martyrs, Grenoble, France 
liquid crystal diamagnetic susceptibility, the threshold magnetic field is large. In an effort to enhance the magnetic susceptibility of liquid crystals, the idea of doping them with fine magnetic particles was theoretically introduced by Brochard and de Gennes [2]. These dilute suspensions of magnetic particles based on nematic liquid crystals are called ferronematics. They have been intensively investigated in recent years both experimentally and theoretically [3-16]. The influence of the magnetic field on ferronematics depends on the coupling energy and mutual orientation of magnetic particles and liquid crystal molecules. The Brochard and de Gennes [2] theory predicted a rigid anchoring with $\boldsymbol{m} \| \boldsymbol{n}$, where the unit vector $\boldsymbol{n}$ (director) denotes the preferential direction of the nematic molecules and the unit vector $\boldsymbol{m}$ denotes orientation of the magnetic moment of the magnetic particles. Later experiments with thermotropic ferronematics excluded the presence of parallel orientation of $\boldsymbol{m}$ and $\boldsymbol{n}$. So, Burylov and Raikher's theory was constructed [3-5]. This theory considers the finite value of the surface density of the anchoring energy $W$ at the nematic-magnetic particle boundary. The finite value of $W$, as well as the parameter $\omega$ that is defined as a ratio of anchoring energy to elastic energy of a liquid crystal $(\omega=W d / K$, where $d$ is the size of the magnetic particles and $K$ is the orientational-elastic Frank modulus), characterize the type of anchoring of nematic molecules on a magnetic particle surface. The parameter $\omega \gg 1$ characterizes rigid anchoring. Soft anchoring is characterized by the parameter $\omega \leq 1$, and unlike rigid anchoring, permits both types of boundary conditions ( $\boldsymbol{m} \| \boldsymbol{n}$ and $\boldsymbol{m} \perp \boldsymbol{n}$ ). Thus, the Burylov and Raikher theory could be applied for thermotropic ferronematics. In its framework the instabilities of the uniform texture in ferronematics exposed to an external magnetic or electric field (Freedericksz transitions) [3-5] could be studied, and the expressions for their critical fields in different geometries have been derived.

In the study of Kedziora et al. [17], the coexistence of nematic and isotropic phases was observed in the 6CHBT liquid crystal dissolved in a nonpolar medium (benzene) in the vicinity of the temperature of the isotropic-to-nematic transition. Due to the existence of a short-range orientational order of the mesogenic molecules 4-(trans4'-n-hexylcyclohexyl)-isothiocyanatobenzene (6CHBT), the pseudonematic domains (droplets of $500 \mathrm{~nm}$ to $1000 \mathrm{~nm}$ in size depending on the temperature) were formed in the isotropic phase. The size of these droplets increased as the temperature of the liquid decreased. The phase diagram of the mixture of 6CHBT and benzene was determined with a polarizing microscope. These results have inspired us to perform similar experiments with the nematogenic liquid crystal 6CHBT dissolved in phenyl isocyanate (that is more stable than benzene) doped with spherical-shaped magnetic nanoparticles [18]. In this study, structural transitions in the liquid crystal 6CHBT dissolved in phenyl isocyanate and doped with nanorods or chain-like magnetic nanoparticles were investigated.

\section{Experiment}

Magnetic nanorods were synthesized through hydrolysis of $\mathrm{FeCl}_{3}$ and $\mathrm{FeSO}_{4}$ solutions (molar ratio $\mathrm{Fe}^{3+} / \mathrm{Fe}^{2+}$ was 2:1) containing urea. The morphology and size distribution (Fig. 1) of the prepared nanorods were measured by transmission electron 

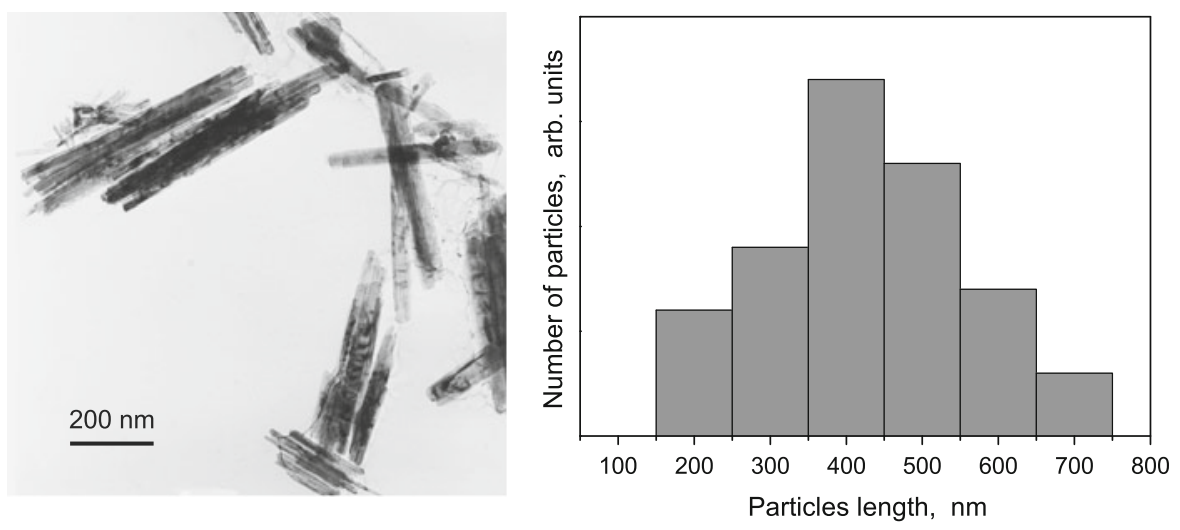

Fig. 1 TEM image (left) and histogram (right) of rod-like particles
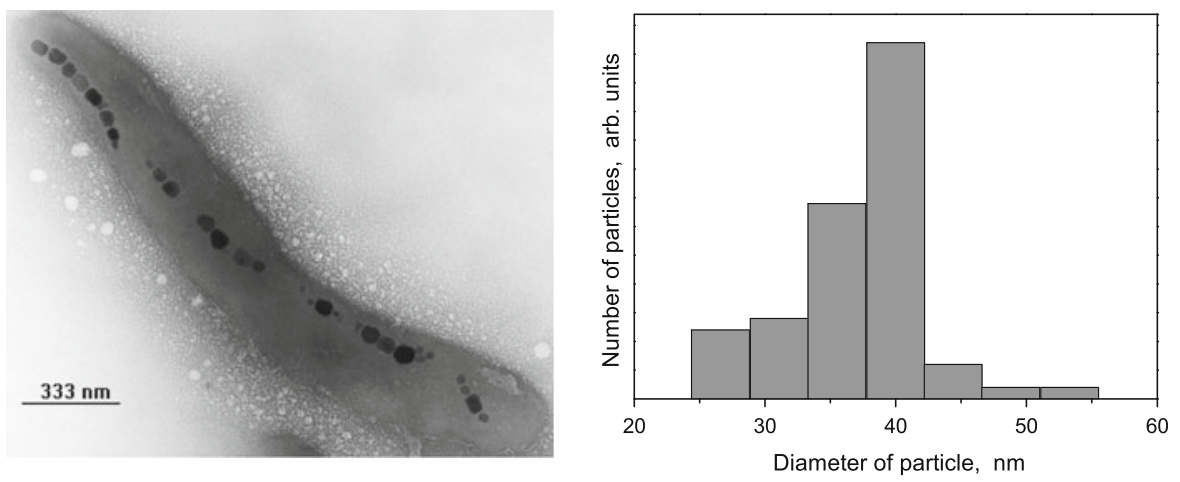

Fig. 2 TEM image of magnetotactic bacteria with magnetosomes (left) and histogram of magnetosomes obtained from magnetotactic bacteria (right)

microscopy (TEM). The average diameter of the nanorods was $20 \mathrm{~nm}$, and the mean length determined from a histogram of size distribution was $400 \mathrm{~nm}$.

The chain-like particles have been obtained from the magnetotactic bacteria magnetotacticum magnetospirillum (AMB-1) [19]. The morphology and size distribution of the obtained magnetosomes were measured by TEM. Figure 2 shows a TEM image of magnetotactic bacteria with magnetosomes and a size distribution of magnetosomes. The mean size of a single magnetic particle coated with surfactant, i.e., of the magnetosome was $36 \mathrm{~nm}$, and the mean length of the chain as a whole was $400 \mathrm{~nm}$. The magnetic properties of nanorods and magnetosomes were investigated by a SQUID magnetometer (Quantum Design MPMS 5XL).

The studied ferronematic samples were based on the thermotropic nematic 6CHBT. The 6CHBT is a low-melting enantiotropic liquid crystal with high chemical stability [20]. The temperature of the nematic-to-isotropic transition (clearing point) of the studied nematic is $T_{N-I}=42.8^{\circ} \mathrm{C}$. The critical magnetic field of pure 6CHBT is $2.63 \mathrm{~T}$. The doping was done by adding nanoparticles, under continuous stirring, to the liquid crystal in the isotropic phase. The volume concentration of the magnetic 


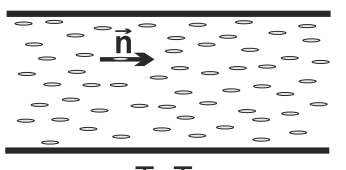

$\mathrm{T}<\mathrm{T}_{\mathrm{N}-1}$

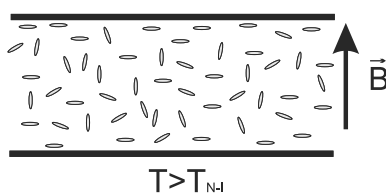

$T>T_{N-1}$

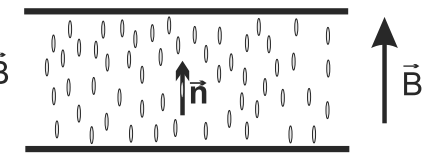

$\mathrm{T}<\mathrm{T}_{\mathrm{N}-1}$

Fig. 3 Cross section of the cell in the initial state, after heating to the temperature $T>T_{N-I}$ and after cooling to the temperature $T<T_{N-I}$ in a magnetic field higher than the critical magnetic field. The magnetic field was applied perpendicular to the electrode surfaces

particles was $5 \times 10^{-4}$. The homogeneity and stability of the samples were verified by optical microscopy and by dielectric measurements indirectly [14]. The formation of droplets of the nematic phase in the isotropic phase was achieved near the isotropic-nematic transition temperature, in the 6CHBT dissolved in phenyl isocyanate and in the 6CHBT dissolved in phenyl isocyanate and doped with nanorods and chainlike magnetic particles, respectively. The structural transitions in the prepared samples were indicated by capacitance measurements in a capacitor made of ITO-coated glass electrodes (LINCAM Co.). The capacitor with the electrode area approximately $1 \mathrm{~cm} \times 1 \mathrm{~cm}$ was connected to a regulated thermostat system; the temperature was stabilized with an accuracy of $0.05^{\circ} \mathrm{C}$. The distance between the electrodes (sample thickness) was $D=5 \mu \mathrm{m}$. The capacitance was measured at the frequency $1 \mathrm{kHz}$ by a high-precision capacitance bridge Andeen Hagerling.

In the experiment, the liquid crystal had an initial planar alignment; i.e., the director was parallel to the capacitor electrodes (see Fig. 3). It should be pointed out for the next consideration that a minimal value of capacitance is observed for planar alignment while a maximal value for homeotropic alignment, i.e., when the director $\boldsymbol{n}$ is perpendicular to the capacitor electrodes. Then the samples were heated to the isotropic phase (approximately $35^{\circ} \mathrm{C}$ ). An external magnetic field was applied perpendicular to the electrode surfaces when samples were in the isotropic phase. Then the samples were cooled at a rate of $2{ }^{\circ} \mathrm{C} \cdot \mathrm{min}^{-1}$ in the external magnetic field. The dependence of the measured capacitance on the external field reflects the re-orientation of the nematic molecules. The stability of the samples in the strong magnetic fields was verified by repeating the capacitance measurements after 5 months on the same samples, with reproducible results.

\section{Results}

Nematic droplets of 6CHBT in the isotropic phase were created in the 6CHBT liquid crystal dissolved in phenyl isocyanate and in the 6CHBT liquid crystal dissolved in phenyl isocyanate and mixed with a small volume concentration of magnetic particles $\phi=5 \times 10^{-4}$, near the isotropic-to-nematic transition temperature. The magnetic particles used for doping were of two different shapes: nanorods and chain-like. Figure 4 shows droplets of the nematic phase of 6CHBT in the isotropic phase, created in the 6CHBT dissolved in phenyl isocyanate, observed under a polarizing microscope. The sample was deposited between two glass slides heated above the nematic-to-isotropic transition point and then slowly cooled. When the 

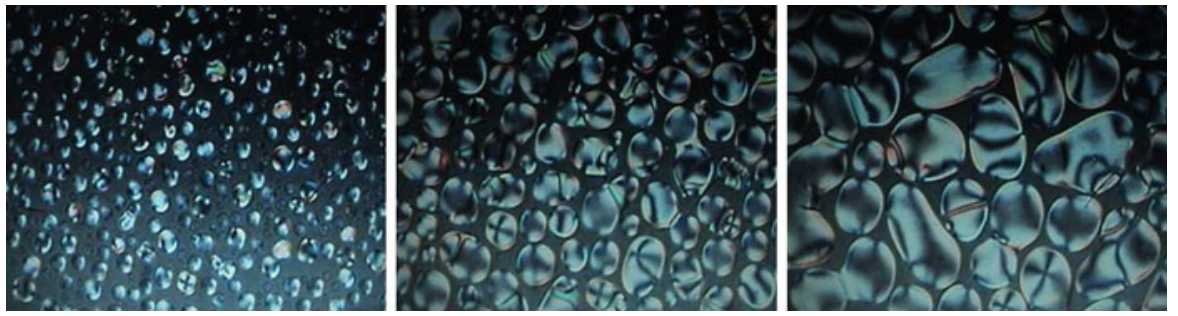

Fig. 4 Formation of the nematic droplets in the isotropic phase in 6CHBT mixed with phenyl isocyanate (molar fraction $X=0.906$ ) at temperatures $24^{\circ} \mathrm{C}, 23^{\circ} \mathrm{C}$, and $22^{\circ} \mathrm{C}$ (from left to right)

temperature is decreased, micro-droplets occur, and as the temperature decreases, the size of droplets increases until the whole sample becomes nematic. A similar behavior was observed also in the case of samples doped with either nanorods or chain-like magnetic particles.

In our previous study [14], from measurements of the 6CHBT-based ferronematics, we have obtained the parameter $\omega=10^{-2}$, which characterizes soft anchoring, and the boundary condition between $\boldsymbol{m}$ and $\boldsymbol{n}$ was found to be parallel. These results are in good agreement with Burylov and Raikher's theory [3-5]. However, our experiments on the droplets indicated that the orientation of the nematic molecules in the droplets could be more complicated than in the case of the pure nematic [18]. The uniform alignment of a liquid crystal matrix is distorted by the inclusion of particles due to the local constrains imposed by the anchoring of the liquid crystal molecules at the surfaces of the magnetic nanoparticles. These constrains can impose conflicting orientations to the liquid crystal, which results in the formation of local defects. These defects depend on the shape and size of the particles and lead to a different behavior of ferronematic samples [21].

Figures 5, 6, and 7 show the temperature dependencies of the capacitance in pure 6CHBT dissolved in phenyl isocyanate (molar fraction of liquid crystal was $X=$ 0.906), and in the 6CHBT liquid crystal dissolved in phenyl isocyanate doped with magnetic nanorods and chain-like magnetic particles, respectively. The temperature dependencies of the capacitances were measured first without a magnetic field $(B=$ $0 \mathrm{~T}$ ) and then after application of magnetic fields of $1 \mathrm{~T}, 2 \mathrm{~T}, 3 \mathrm{~T}, 4 \mathrm{~T}, 6 \mathrm{~T}, 8 \mathrm{~T}$, and $12 \mathrm{~T}$. The samples were first heated to the isotropic phase, then a magnetic field was applied, and samples were slowly cooled to the nematic phase.

As seen from Figs. 5, 6, and 7 for the magnetic fields $1 \mathrm{~T}, 2 \mathrm{~T}$, and $3 \mathrm{~T}$, as the temperature reaches a value at which the droplets of the nematic phase in the isotropic phase occur, the capacitance of all samples decreases with decreasing temperature, i.e., the applied magnetic field is not strong enough to turn the molecules in droplets to its direction. For $B>4 \mathrm{~T}$, the applied magnetic field is strong enough to rotate the director to its direction, i.e., the liquid crystal molecules are oriented perpendicular to the electrodes (the capacitance increases with decreasing $T$ ). However, for pure 6CHBT at $B=2 \mathrm{~T}$ the capacitance does not reach the minimal value (the value of the capacitance at $B=0$ ) also at $T=14{ }^{\circ} \mathrm{C}$. In the case of samples doped with magnetic nanoparticles, the same behavior is observed at a magnetic field $B=3 \mathrm{~T}$. In the case 


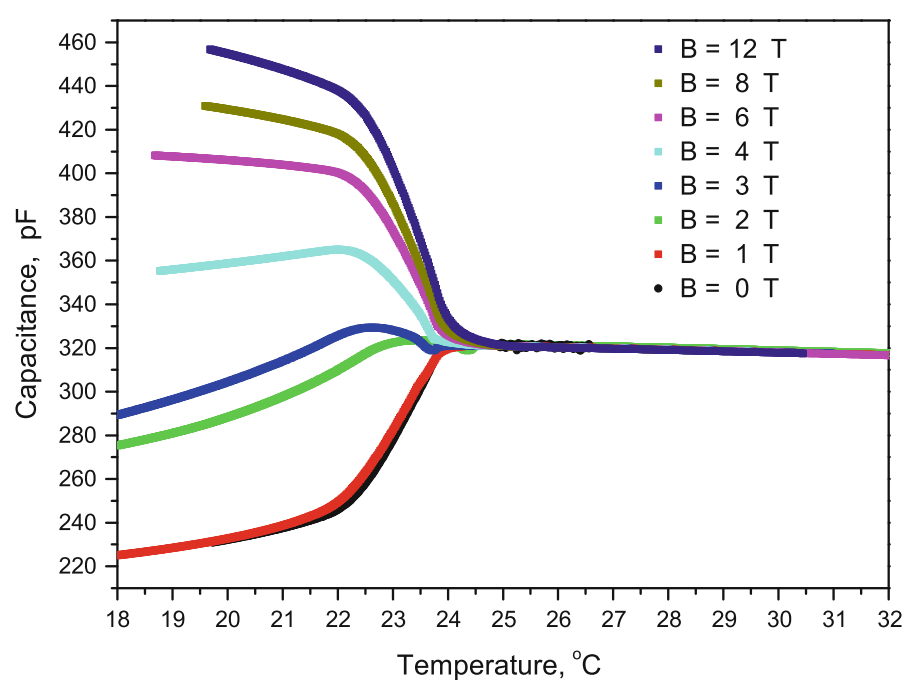

Fig. 5 Temperature dependences of the capacitance of 6CHBT dissolved in phenyl isocyanate

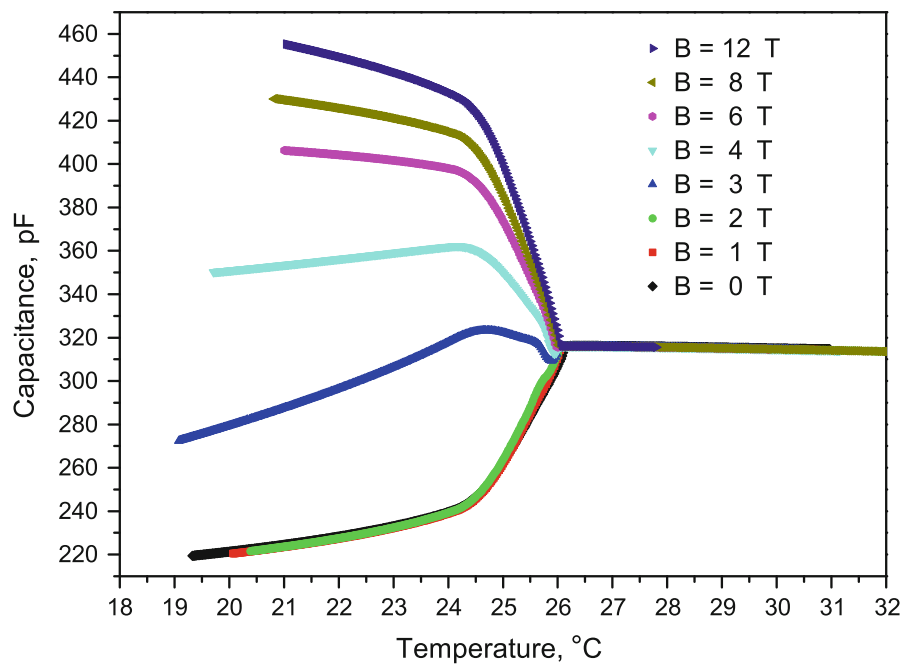

Fig. 6 Temperature dependences of the capacitance of 6CHBT dissolved in phenyl isocyanate doped with magnetic nanorods

of doping with spherical magnetic particles, a decrease of the critical magnetic field due to doping was observed [18]. We assumed a similar effect would be observed due to doping with magnetic nanorods as well as with chain-like magnetic particles. However, as one can see from Figs. 6 and 7, in the case of doping with magnetic nanorods and chain-like magnetic particles, more complicated behavior is observed. While in the case of a sample without doping at a magnetic field of $2 \mathrm{~T}$, the competition between planar and homeotropic orientation is observed; for the doped samples, a magnetic field of $2 \mathrm{~T}$ has no influence on the orientation of the nematic molecules. 


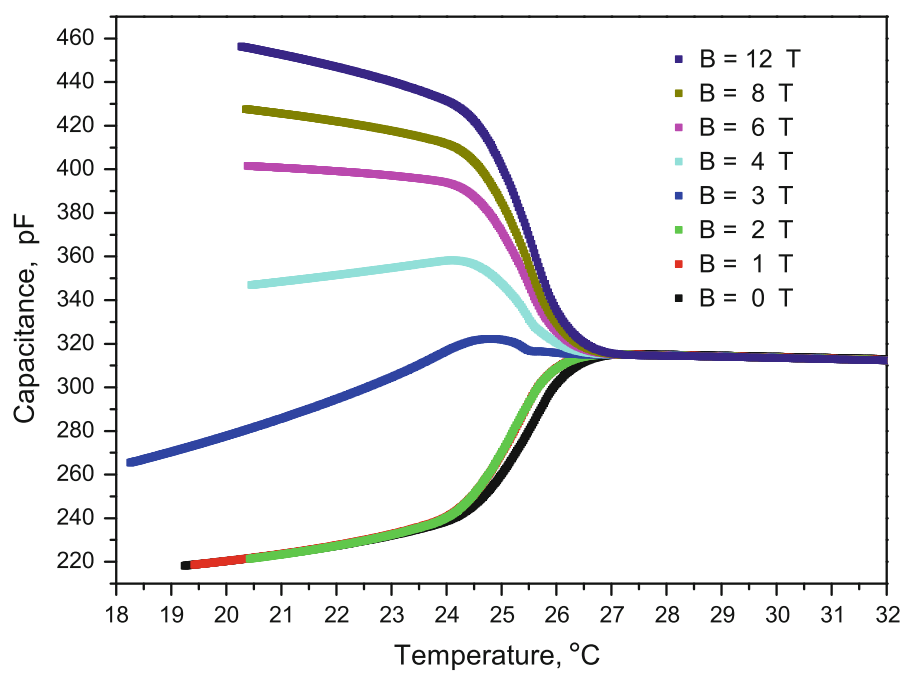

Fig. 7 Temperature dependences of the capacitance for 6CHBT dissolved in phenyl isocyanate doped with chain-like magnetic particles

In our previous study [18], an increase of the temperature of the isotropic-to-nematic phase transition via a droplet state due to doping the 6CHBT liquid crystal with spherical magnetic particles was observed. A similar behavior was observed also in the samples doped with magnetic nanorods as well as with chain-like magnetic particles. The increase in the temperature of the isotropic-to-nematic phase transition via a droplet state is shown in Fig. 8 and is summarized in Table 1. Moreover, the increase of the capacitance of the sample doped with nanorods is very sharp.

The obtained results suggest the following possible explanation. In the case of doping with spherical particles, we assumed that the interaction between the magnetic particles and the liquid crystal molecules favors a parallel initial orientation of the magnetic moments of the magnetic particles and the director around them [18]. A similar situation is also seen in the case of doping with the nanorods and the chain-like particles. The mean size of the spherical particles was $10 \mathrm{~nm}$, while the mean size of the nanorods and chains of magnetosomes was $400 \mathrm{~nm}$. As was shown in previous studies $[9,16,21]$, the particle size and functionalization of the particles play an important role in the coupling between the magnetic moment of the magnetic particles and the director of the liquid crystal. The obtained results indicate that orientation of the magnetic moment and the director in both the cases (nanorods and chain-like particles) is parallel. In the nematic phase, the preferred orientation of molecules is parallel to the surface of the electrodes due to the surface treatment. We suppose the orientation of the magnetic moment of the magnetic particles and the director is parallel, and so the magnetic moment of the magnetic particles is oriented parallel to the surface of the electrodes as shown in Fig. 9. As the sample is heated to a temperature higher than $T_{N-I}$, the sample is in the isotropic phase and the molecules as well as the magnetic moments are disordered. The applied magnetic field stabilizes the orientation of the magnetic particles. For magnetic fields lower than the critical, as the temperature is 


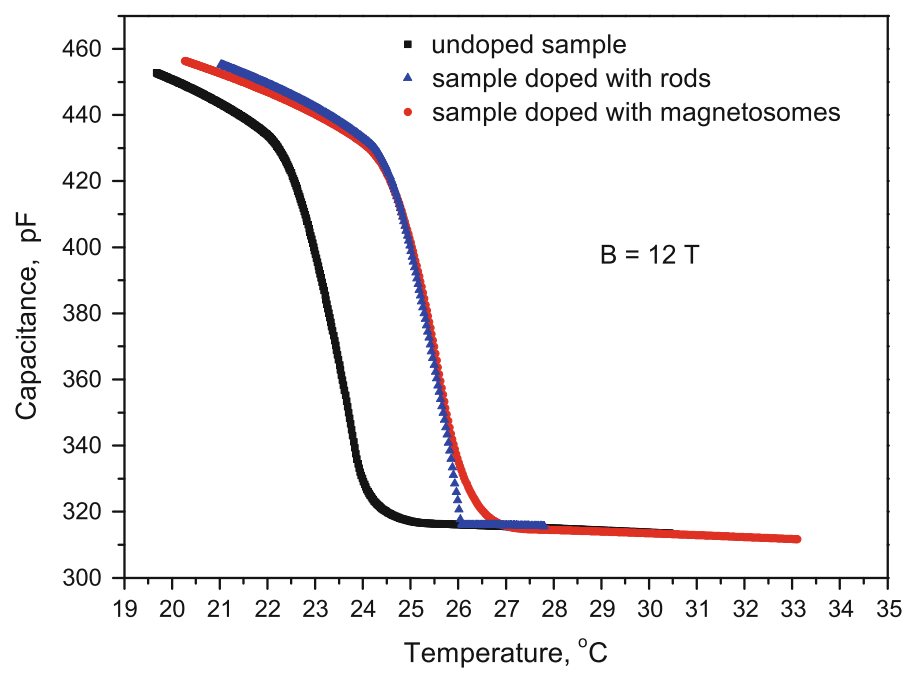

Fig. 8 Temperature dependences of the capacitance of 6CHBT dissolved in phenyl isocyanate without magnetic particles and doped with magnetic nanorods and chain-like magnetic particles at an applied magnetic field of $12 \mathrm{~T}$

Table 1 Temperatures of the isotropic-to-nematic phase transition for 6CHBT dissolved in phenyl isocyanate without magnetic particles and doped with magnetic nanorods and chain-like magnetic particles

\begin{tabular}{ll}
\hline Sample & Temperature $\left({ }^{\circ} \mathrm{C}\right)$ \\
\hline 6 CHBT & 24.55 \\
6 CHBT with nanorods & 26.05 \\
6 CHBT with magnetosomes & 26.80 \\
\hline
\end{tabular}

decreasing, droplets of the nematic phase in the isotropic phase occur. The orientation of the director inside the droplets is random at close to $T_{N-I}$; in the two-phase region, the nematic droplets are surrounded by the isotropic phase, so the influence of the surfaces are negligible. As the temperature decreases and the droplets grow and develop contact with the electrodes, the interaction with the aligning surfaces becomes more effective and finally the director turns back towards the direction parallel to the electrodes, i.e., the capacitance decreases with decreasing temperature. For magnetic fields higher than the critical magnetic field, the director inside the droplets is oriented to the direction of the applied magnetic field, i.e., the capacitance increases with decreasing temperature. Moreover, the magnetic nanoparticles play the role of the walls (the size of magnetic nanoparticles is several orders bigger than the size of liquid crystal molecules) that tend to align the molecules of the liquid crystal. Thus, a nematic order is induced by the presence of the magnetic nanoparticles already above $T_{N-I}$, i.e., the isotropic-nematic phase transition is apparently shifted to higher temperatures.

The competition between the influences of the electrode surfaces and the external electric field results in another interesting phenomenon near $T_{N-I}$ as depicted in Figs. 10, 11, and 12. As one can see from these figures, the competition is more 


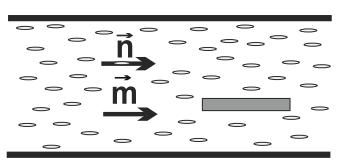

$\mathrm{T}<\mathrm{T}_{\mathrm{N}-\mathrm{I}}$

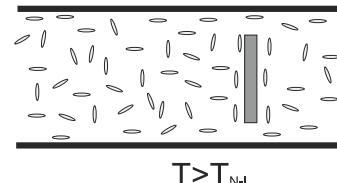

$\mathrm{T}>\mathrm{T}_{\mathrm{N}-1}$

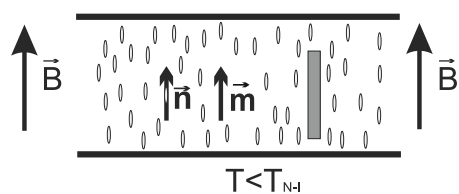

$\mathrm{T}<\mathrm{T}_{\mathrm{N}-1}$

Fig. 9 Orientation of the ferronematic in the cell in the initial state, after heating to the temperature $T>T_{N-I}$ and after cooling to the temperature $T<T_{N-I}$ in a magnetic field higher than the critical magnetic field

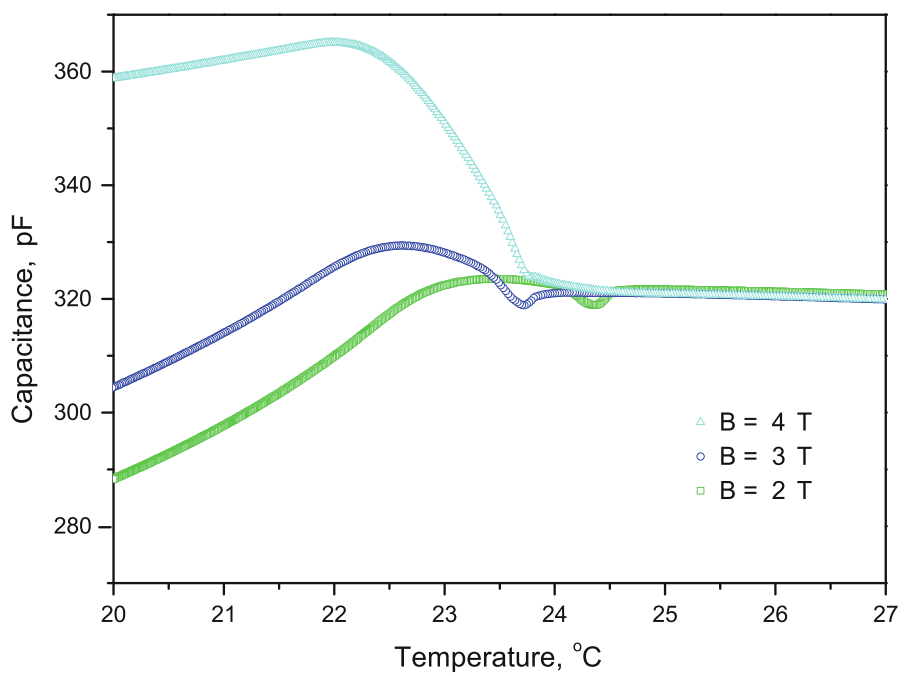

Fig. 10 Temperature dependences of the capacitance of 6CHBT dissolved in phenyl isocyanate at magnetic fields of $2 \mathrm{~T}, 3 \mathrm{~T}$, and $4 \mathrm{~T}$

significant in the case of the sample without magnetic particles. In the nematic phase, the director is preferably oriented parallel to the surface of the electrodes due to the surface treatment. However, close to $T_{N-I}$ in the two-phase region, the nematic droplets are surrounded by the isotropic phase, so the influence of surfaces are negligible. As a consequence, the magnetic field can turn the director, inside of the created nematic droplets to its direction. As the temperature decreases and the droplets grow and develop contact with the electrodes, the interaction with the aligning surfaces becomes more effective and finally the director turns back towards the direction parallel to the electrodes (see the change in the slope in Figs.10, 11, 12). A similar situation is also observed in samples doped with the magnetic particles. Microscopic observation under a magnetic field would be required to justify this assumption.

\section{Conclusion}

Embedding magnetic particles in a nematic liquid crystal causes an effective orientational coupling between the magnetic moment of the magnetic particles and the director of the nematic. This coupling may come from the anisotropy of anchoring 


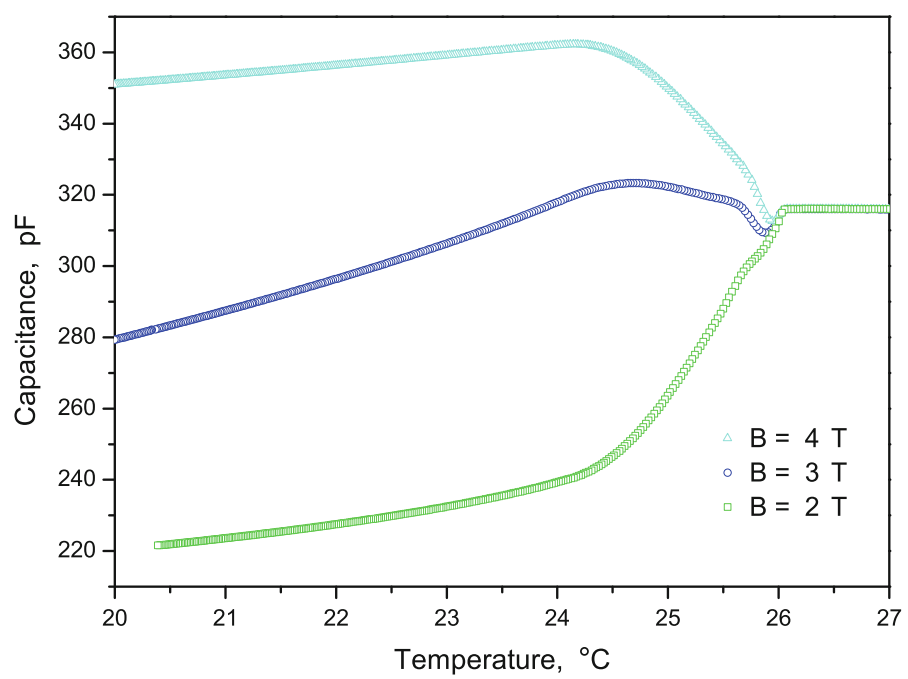

Fig. 11 Temperature dependences of the capacitance for 6CHBT dissolved in phenyl isocyanate doped with magnetic nanorods at magnetic fields of $2 \mathrm{~T}, 3 \mathrm{~T}$, and $4 \mathrm{~T}$

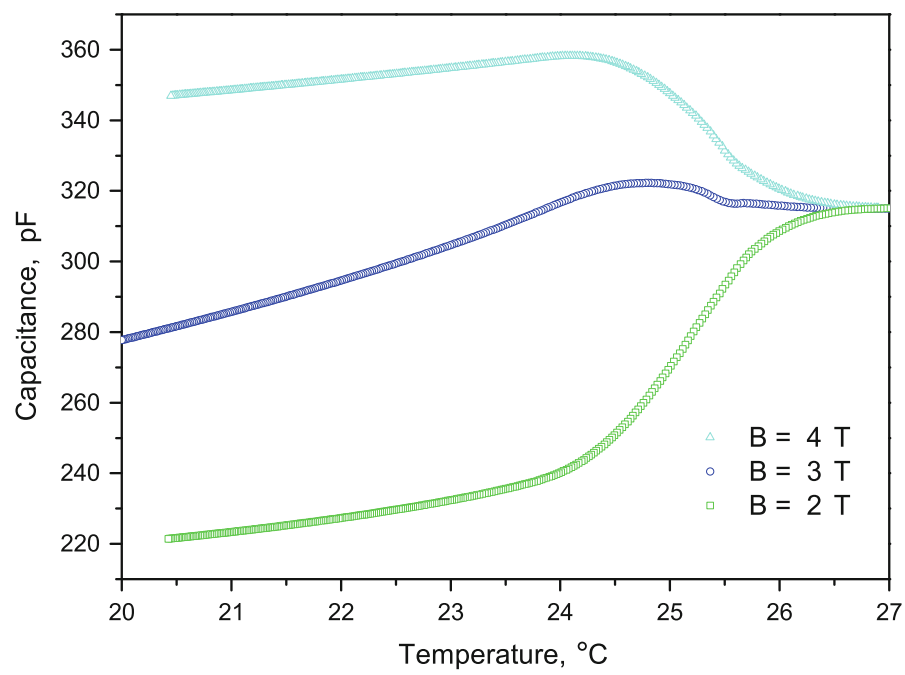

Fig. 12 Temperature dependences of the capacitance for 6CHBT dissolved in phenyl isocyanate doped with chain-like magnetic particles at magnetic fields of $2 \mathrm{~T}, 3 \mathrm{~T}$, and $4 \mathrm{~T}$

of the nematic molecules on the particle surface. The obtained results suggest that in the case of ferronematics based on the 6CHBT liquid crystal dissolved in phenyl isocyanate and doped with magnetic nanorods and chain-like magnetic particles, the anchoring of the liquid crystal molecules on the surface of magnetic particles is soft and their orientation is parallel to the magnetic moment. Moreover, the temperature of the phase transition increases due to doping with magnetic particles due to a 
field-induced nematic order. Future studies will focus on the phase transitions in combined electric and magnetic fields.

Acknowledgments This study was supported by the Slovak Academy of Sciences Grant VEGA No. 0077 and Center of Excellence Nanofluid, Slovak Research and Development Agency under contract No. APVV-0509-07 and contract No. SK-HU-0008-08, the Grenoble High Magnetic Field Laboratory, with support of EC from the 7th FP capacities "Transnational Access"-Contract No. 228043-EuroMagNET II, and with support by European Regional Development Fund Projects 26220120021 and 26220120033.

Open Access This article is distributed under the terms of the Creative Commons Attribution Noncommercial License which permits any noncommercial use, distribution, and reproduction in any medium, provided the original author(s) and source are credited.

\section{References}

1. P.G. Gennes, J. Prost, The Physics of Liquid Crystals (Clarendon Press, Oxford, 1993)

2. F. Brochard, P.G. Gennes, J. Phys. Paris 31, 691 (1970)

3. S.V. Burylov, Y.L. Raikher, J. Phys. Lett. A 149, 279 (1990)

4. S.V. Burylov, Y.L. Raikher, J. Magn. Magn. Mater. 122, 62 (1993)

5. S.V. Burylov, Y.L. Raikher, Mol. Cryst. Liq. Cryst. 255, 107 (1995)

6. S.V. Burylov, V.I. Zadorozhnii, I.P. Pinkevich, V.Yu. Reshetnyak, T.J. Sluckin, J. Magn. Magn. Mater. 252, 153 (2002)

7. D.V. Makarov, A.N. Zakhlevnykh, J. Magn. Magn. Mater. 320, 1312 (2008)

8. C. Satiro, Phys. Rev. E 80, 042701 (2009)

9. L.J. Martinez-Miranda, L.K. Kurihara, J. Appl. Phys. 105, 084305 (2009)

10. E. Petrescu, E.R. Bena, J. Magn. Magn. Mater. 321, 2757 (2009)

11. J.C. Bacri, A.M. Figueiredo Neto, Phys. Rev. E 50, 3860 (1994)

12. A.N. Zakhlevnykh, P.A. Sosnin, J. Magn. Magn. Mater. 146, 103 (1995)

13. P. Kopčanský, I. Potočová, M. Koneracká, M. Timko, A.G.M. Jansen, J. Jadzyn, G. Czechovski, J. Magn. Magn. Mater. 289, 101 (2005)

14. P. Kopčanský, M. Koneracká, M. Timko, I. Potočová, L. Tomčo, N. Tomašovičová, V. Závišová, J. Jadzyn, J. Magn. Magn. Mater. 300, 75 (2006)

15. N. Tomašovičová, M. Koneracká, P. Kopčanský, M. Timko, V. Závišová, J. Jadzyn, Phase Trans. 79, 595 (2006)

16. P. Kopčanský, N. Tomašovičová, M. Koneracká, V. Závišová, M. Timko, A. Džarová, A. Šprincová, N. Éber, K. Fodor-Csorba, T. Tóth-Katona, A. Vajda, J. Jadzyn, Phys. Rev. E 78, 011702 (2008)

17. P. Kedziora, J. Jadzyn, L. Hellemans, Phys. Rev. E 66, 021709 (2002)

18. N. Tomašovičová, P. Kopčanský, M. Koneracká, L. Tomčo, V. Závišová, M. Timko, N. Éber, K. Fodor-Csorba, T. Tóth-Katona, A. Vajda, J. Jadzyn, J. Phys. Condens. Matter 20, 204123 (2008)

19. M. Timko, A. Džarová, V. Závišová, M. Koneracká, A. Šprincová, P. Kopčanský, J. Kováč, I. Vavra, A. Szlaferek, Magnetohydrodynamics 44, 113 (2008)

20. R. Dabrowski, J. Dziaduszek, T. Szczucinski, Mol. Cryst. Liq. Cryst. Lett. 102, 155 (1984)

21. F.R. Hung, Phys. Rev. E 79, 021705 (2009) 